\title{
Topographical Evolution of Lead Zirconate Titanate (PZT) Thin Films Patterned by Micromolding in Capillaries
}

\author{
Christopher R. Martin and Ilhan A. Aksay* \\ Department of Chemical Engineering, Princeton University, Princeton, New Jersey 08544-5263
}

Received: January 9, 2003

\begin{abstract}
The patterning of sol-gel-derived thin films by micromolding in capillaries can produce unintended topographical deviations from the shape of the original mold that may limit the utility of the technique in potential applications. During drying and heat treatment, nonuniform shrinkage across the film due to the densification of the gel matrix results in "double-peak" film topographies whereby the film thickness is greater at the lateral edges than in the middle. Using the same framework used to understand the imbibition and wetting of the sol-gel in the capillary channels, we developed a mechanism to explain the formation of the double-peak profile. As a model system, patterned $\mathrm{Pb}\left(\mathrm{Zr}_{0.52} \mathrm{Ti}_{0.48}\right) \mathrm{O}_{3}$ thin films were studied. Atomic force microscopic characterization was used to quantify the effect of the rate of gelation on the topography of the patterned thin films. Modifications to the channel mold design eliminate the peak formation, producing more homogeneous patterns that better replicate the features of the mold.
\end{abstract}

\section{Introduction}

Considerable research effort has been focused on the development of thin film technologies for ferroelectric materials. ${ }^{1-5}$ Ferroelectric materials, such as lead zirconate titanate $\left(\mathrm{Pb}(\mathrm{Zr}, \mathrm{Ti}) \mathrm{O}_{3}, \mathrm{PZT}\right)$, can be used in integrated electronic devices such as capacitors, transducers, pyroelectric detectors, thermistors, and dielectrics. ${ }^{1}$ A growing field of study is the integration of patterned piezoelectric thin films in microelectromechanical systems (MEMS). Traditional silicon-based integrated circuit (IC) manufacturing methods and materials are combined with specialized processes, novel materials, and customized packaging techniques to fabricate MEMS capable of sensing, actuating, and processing information. MEMS may include such fabricated devices as accelerometers, infrared devices, flow meters, pumps, and motors ranging in sizes from the micrometer to centimeter length scales. ${ }^{2}$ MEMS have found applications in the biomedical field as blood pressure sensors, ${ }^{3}$ information processing as displays, ${ }^{4}$ and the automotive industry as accelerometers for deploying air bags. ${ }^{5}$ A host of new microactuator and microsensing applications can be realized by successfully incorporating the superior piezoelectric and pyroelectric properties of inorganic ferroelectric materials into MEMS devices.

Currently, nearly all microelectronic devices and IC architectures are fabricated using subtractive processing methods. ${ }^{6}$ Most deposition methods, such as spin- and dip-coating, produce continuous thin films across the entire surface of the substrate. The various device features are defined in the thin film layer by selectively etching away the unwanted material leaving behind the desired microstructure. The subtractive processing steps usually require complex and costly lithographic patterning steps, followed by wet chemical etching or reactive ion etching (RIE). ${ }^{7}$ These methods have worked very well for traditional silicon-based IC manufacturing but are of limited utility to proposed thin film materials such as inorganic ferroelectrics. Depending on the material of interest, these methods may require highly controlled environments, etch anisotropically, or leave undesirable residual chemical artifacts. In the case of PZT thinfilms, subtractive patterning requires dedicated RIE equipment to minimize lead contamination. ${ }^{8}$

The successful implementation of thin film ferroelectric materials in micrometer scale structures requires new additive patterning techniques that are compatible with existing technologies. To minimize production costs, these processes must be able to replicate patterns over large areas with high throughput. The methods should not require investment in new, expensive, dedicated capital equipment to be attractive to potential manufacturers. The use of the soft lithography techniques pioneered by Whitesides and co-workers ${ }^{9}$ has resulted in the development of new fabrication methods for micropatterned ceramic thin films. ${ }^{8,10-20}$

The techniques used for patterning inorganic thin films fall into three categories. The first uses the elastomeric mold as a stamp to effect the microcontact printing $(\mu \mathrm{CP})$ of selfassembled monolayers (SAM) of surfactants, most commonly octadecyltrichlorosilane (OTS). ${ }^{21}$ The patterned SAM layer is then used as a template for the selective deposition of the metal oxide thin film. A chemical precursor solution is spin-coated on top of the substrate with a patterned SAM. After heat treatment between 300 and $400{ }^{\circ} \mathrm{C}$, the regions of the film deposited on top of the SAM show poor adhesion and crack because of the pyrolysis of the underlying organic ligand molecules. This material is removed with mild mechanical abrasion using a cotton felt dampened with 2-propanol. A higher-temperature heat treatment is then used to remove any remaining organic components and convert the remaining precursor to the desired crystal structure..$^{8,10}$

Jeon et al. ${ }^{10}$ first reported the use of $\mu \mathrm{CP}$ to pattern a SAM from OTS in hexanol as a template for depositing lithium niobate $\left(\mathrm{LiNbO}_{3}\right)$ and lead lanthanum titanate $\left(\mathrm{Pb}_{1-3 x / 2} \mathrm{La}_{x} \mathrm{TiO}_{3}\right)$ on sapphire, silicon, and indium tin oxide substrates. Later work by the same research group demonstrated the use of the technique for patterning tantalum $(\mathrm{V})$ oxide $\left(\mathrm{Ta}_{2} \mathrm{O}_{5}\right)$ on silicon, aluminized silicon, and platinized silicon wafers ${ }^{11}$ and PZT on platinum. ${ }^{12}$ They achieved line widths as small as $4 \mu \mathrm{m}$ with 
$100 \mathrm{~nm}$ resolution and vertical thickness of 80 to $120 \mathrm{~nm}$ with their resulting patterned thin film oxides. ${ }^{11}$ Their work is well summarized in a review article. ${ }^{8}$ A variation of the technique has been used by Bechiniger et al. ${ }^{22}$ to pattern an array of 150 $\mathrm{nm}$ tungsten oxide dots using a poly(dimethylsiloxane) (PDMS) stamp cast from a self-assembled, hexagonally arranged, closepacked monolayer of $600 \mathrm{~nm}$ diameter polystyrene colloidal spheres. The SAM was patterned from a combination of octadecanethiol and 11-mercaptoundecanol on gold-coated glass sheets and then dip-coated in the tungsten alkoxide precursor solution.

The second method used to pattern ceramics from their chemical precursor is a direct stamping technique called microtransfer molding ( $\mu \mathrm{TM}) .{ }^{13}$ In $\mu \mathrm{TM}$, a prepolymer or chemical precursor solution is deposited into the recessed regions of the patterned side of the stamp, most commonly by spin-coating. The stamp is then brought into contact with the substrate. The low interfacial energy of the PDMS allows for the patterned material to remain on the surface as the stamp is peeled away. ${ }^{9}$ The material is then ready for heat treatment. This technique has been used to pattern spin-on glass and zirconia $\left(\mathrm{ZrO}_{2}\right)$ on a number of substrates ${ }^{13}$ and $\mathrm{SrBi}_{2} \mathrm{Ta}_{2} \mathrm{O}_{9}(\mathrm{SBT})$ lines on a (001) $\mathrm{SrTiO}_{3}$ substrate. ${ }^{14}$ To date, the smallest reported resolution is $500 \mathrm{~nm}$, as reported by Lange and co-workers who patterned lines of monoclinic zirconia $\left(\mathrm{ZrO}_{2}\right)$ orientated epitaxially from a zirconyl 2-ethylhexanoate precursor solution. ${ }^{15}$

The third technique, micromolding in capillaries (MIMIC), ${ }^{23-25}$ has been used to produce ceramic features with dimensions smaller than $500 \mathrm{~nm} .{ }^{18,20}$ MIMIC is a single-step patterning process whereby an elastomeric mold is placed against a flat substrate to produce a network of channels that a liquid precursor fills by capillary suction. Patterned microstructures of oxide materials such as $\mathrm{SiO}_{2},{ }^{16,17} \mathrm{ZrO}_{2}, \mathrm{SnO}_{2},{ }^{18} \mathrm{SrNb}_{2} \mathrm{O}_{7},{ }^{19}$ and $\mathrm{Pb}$ $(\mathrm{Zr}, \mathrm{Ti}) \mathrm{O}_{3}{ }^{19,20}$ have been fabricated from their respective chemical solution precursors.

The primary drawback of additive patterning techniques, such as MIMIC, that use sol-gel precursors is the large nonuniform volumetric shrinkage of the materials during the drying and sintering of the patterned lines from the dimensions of the original mold. ${ }^{18-20}$ Learning to control the evolution of the thin film topography is a critical hurdle that must be overcome for the use of MIMIC and other related techniques in commercial applications. The presence of a highly irregular patterned surface makes it more difficult to later compatibly deposit functionalized coatings, metal contacts, and circuits to complete the device. Typical microfabrication steps of spin-coating of proceeding thin films, evaporation of metal layers, and pattern alignment for photolithography can become problematic with surfaces with sharp peaks and irregular boundaries in the underlying layers. In MEMS, control of the shape of the film is of utmost importance in fabrication. For example, our research is focused on the development of a free-standing piezoelectric microcantilever for biodetection applications. Deviations in the shape of the patterned film from the intended rectangular cross section may result in the introduction of additional resonance modes and blurring of the intended resonance peaks that degrade the device performance.

The work reported in this manuscript evaluates the role of the drying conditions during MIMIC on the topography of patterned sol-gel-derived PZT thin films. Previous studies have neglected to explain the nonuniform topography of the patterned film that can be clearly observed in the published atomic force microscopy (AFM) images. ${ }^{18,19}$ The patterned films have a characteristic "double-peak" profile in which the height of the film at the edges can be nearly twice as thick as that of the film in the middle of the feature. By understanding the origins of this nonuniformity, alteration of the topography of the mold profile can be used to produce more homogeneous thin films. While our experiments solely characterize PZT thin films, the results should be applicable to any material derived from chemical precursor solutions that exhibit significant densification upon conversion to the desired phase.

\section{Experimental Section}

A. Sol-Gel Preparation. The $\mathrm{Pb}\left(\mathrm{Zr}_{0.52} \mathrm{Ti}_{0.48}\right) \mathrm{O}_{3}$ chemical precursor solutions were prepared by using a chelation synthesis sol-gel approach developed by Yi et al. ${ }^{26}$ Lead(II) acetate trihydrate (Aldrich, Milwaukee, WI) was dissolved in glacial acetic acid in a 2:1 mass ratio. The solution was heated to 105 ${ }^{\circ} \mathrm{C}$ for 5-10 min to remove the water from the lead acetate trihydrate. The solution was transferred to a humidity-free glovebox for the addition of stoichiometric amounts of zirconium(IV) propoxide and titanium(IV) isopropoxide (both supplied by Aldrich, Milwaukee, WI). After the solution was stirred for 15-20 min for the chelation reactions to take place, the solution was removed from the glovebox, and distilled water was added to initiate the condensation reactions and promote the formation of the metal-oxide oligomeric chains. The amount of the water addition can be used to adjust the viscosity, but this also impacts the amount of volumetric shrinkage of the sol-gel upon drying and heat treatment.

B. Micromolding in Capillaries. MIMIC, like other soft lithography techniques, begins with the fabrication of masters with relief features that correspond to the desired features on the final patterned surface. Masters were produced by traditional photolithographic microfabrication techniques. The lithographic masters were patterned by a photomask design that produced linear channels with widths of $2,3,5,7,10,15$, and $20 \mu \mathrm{m}$ in close proximity to each other to permit a more systematic evaluation of the effects of different line widths. In this work, the channels on the masters were patterned either in a photoresist (Clariant AZ-5214, Somerville, NJ) thin film layer deposited on the surface of a (100) Si wafer (SiliconQuest International, Santa Clara, CA) or directly in the Si using RIE (PlasmaTherm 790 RIE, Uniaxis USA, Inc., St. Petersburg, FL).

The general procedure for MIMIC is depicted in Figure 1. ${ }^{9,23-25}$ A PDMS mold is cast against the lithographic master by using the commercially available Sylgard 184 kit (Dow Corning, Midland, MI). The liquid prepolymer is mixed in a 10:1 ratio with the initiator and poured on top of the master. The curing polymer is held under vacuum to remove any bubbles introduced during the mixing and improve the fidelity of the pattern transfer. After curing overnight, the PDMS is peeled off of the master and cut so that the microchannels are exposed. The PDMS mold is placed patterned side down on a clean (100) $\mathrm{Si}$ wafer with a native oxide layer and a pool of the PZT solgel is placed along the periphery of the mold. Capillary forces spontaneously draw the sol-gel into the channels where the solution gels and dries forming the patterned thin film.

The rate of drying is controlled by the conditions in which the sample is placed. Fast drying conditions of $3-4 \mathrm{~h}$ to gelation are achieved by allowing the sample to dry in the open air of the laboratory. Sealing the sample in a small Petri dish locks in the humidity from the evaporating water, resulting in slower drying conditions that stretch gelation to periods as long as 48 h. The slower drying conditions permit the sol-gel to infiltrate the channels further, allowing defect-free areas as large as 1 $\mathrm{cm}^{2}$ to be patterned. ${ }^{20}$ 


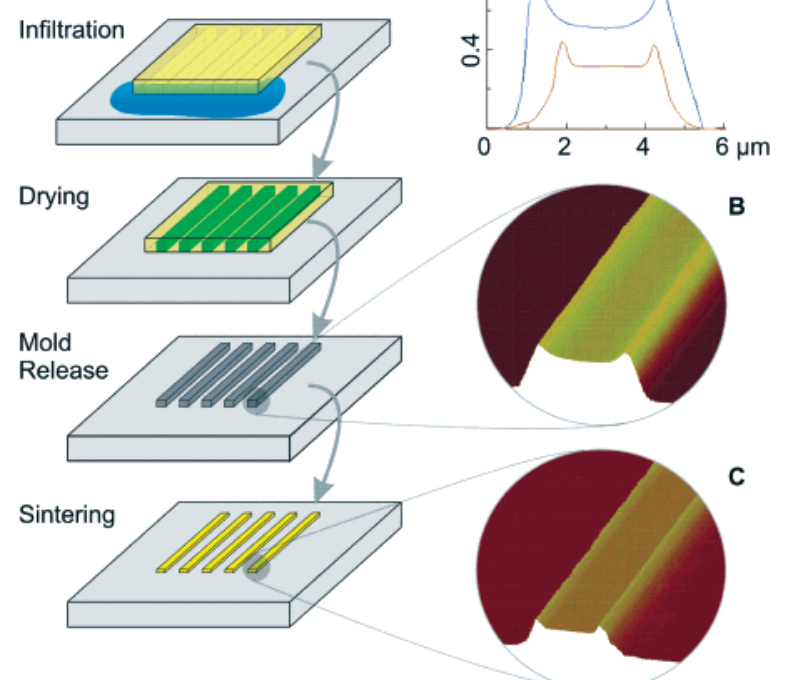

Figure 1. Schematic diagram of micromolding in capillaries to pattern PZT from a sol-gel. The insets show AFM topography images of PZT lines on a (100) silicon wafer patterned by MIMIC having $5 \mu \mathrm{m}$ wide by $1.85 \mu \mathrm{m}$ high channels (B) after drying at room temperature and (C) after heat treatment at $600{ }^{\circ} \mathrm{C}$ for $3 \mathrm{~h}$. In panel A, a cross-section view of the pre- (blue) and postheat treatment (red) lines more clearly shows the evolution of film topography as shrinkage occurs.

Once the sol-gel is dry, the excess material around the periphery of the mold can be scraped off with a razor blade and the PDMS peeled off of the substrate. PDMS is an excellent choice for MIMIC because of its low surface energy ( $\gamma_{\text {PDMS }- \text { air }}$ $=21.6$ dyn $\mathrm{cm}^{-1}$ ), lack of reactivity with the PZT sol-gel, and permeability to air and water vapor., 9,23 These features permit the PZT gel to dry within the confines of the mold and adhere strongly to the Si surface but not to the PDMS channel walls.

C. Heat Treatment. After removal of the PDMS mold, the patterned PZT gel is heat treated in an electric resistance furnace (Thermolyne type 47900 furnace, Dubuque, IA) at $600{ }^{\circ} \mathrm{C}$ for $3 \mathrm{~h}$. The samples are heated using a constant ramp rate of 10 ${ }^{\circ} \mathrm{C} / \mathrm{min}$. The heat treatment permits the volatilization of any remaining organic components of the gel, densification of the film, and conversion to the crystalline perovskite phase. ${ }^{20}$

D. Characterization. The topography of the patterned thin films was examined with an atomic force microscope (Digital Instruments Multimode SPM with Nanoscope IIIa controller, Digital Instruments, Veeco Metrology Group, Santa Barbara, CA) operating in contact mode with oxide-sharpened silicon nitride probes (Digital Instruments NP-S). Quantitative data from the cross sections of the patterned lines was obtained using the section analysis tool from the instrument software. The results were validated by examination with a field emission scanning electron microscope (SEM) (Philips XL30, FEI, Hillsboro, OR). The SEM samples were prepared by coating $20 \AA$ of iridium and examined at 5-10 keV. Both plane view and crosssectioned samples were examined in SEM.

\section{Results and Discussion}

A. Shrinkage. Top-view SEM images of micropatterned lines produced on a Si wafer using $1.85 \mu \mathrm{m}$ high channels are shown in Figure 2 after (A) the removal of the PDMS mold and (B) the heat treatment. The lines were patterned from the mold designed for these experiments that placed different sized lines in close proximity to each other. In these images, the $7,10,15$,
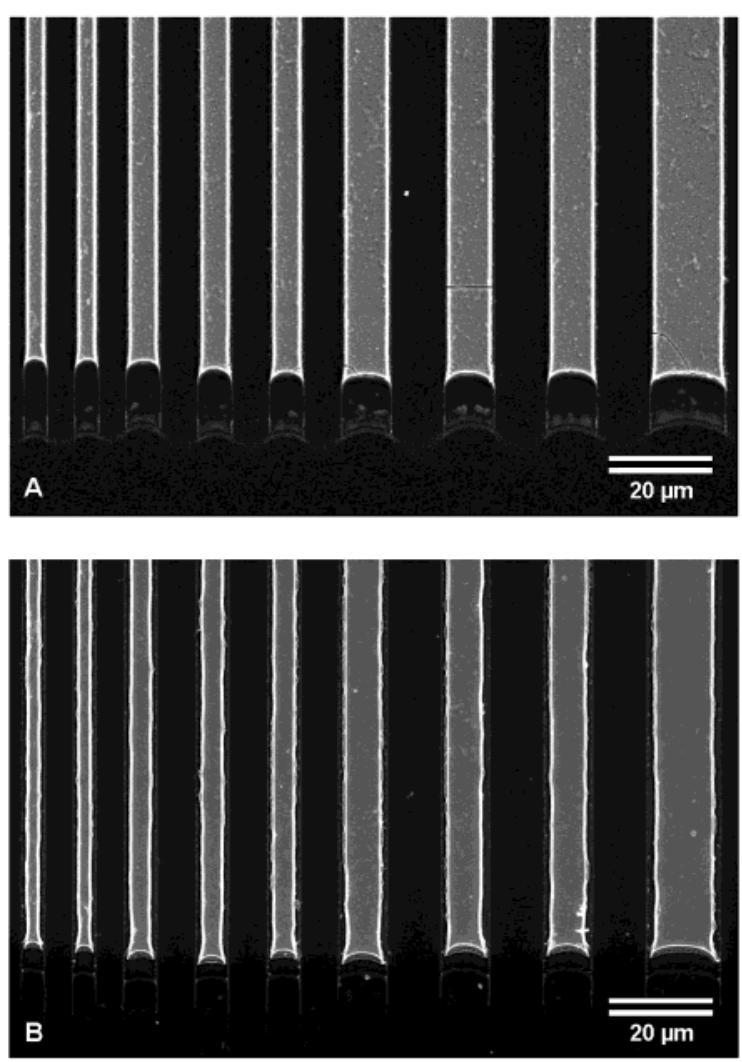

Figure 2. SEM images of PZT lines on a (100) silicon wafer patterned by MIMIC having $1.85 \mu \mathrm{m}$ high channels (A) after drying at room temperature and (B) after heat treatment at $600{ }^{\circ} \mathrm{C}$ for $3 \mathrm{~h}$. The line widths on the mold vary from 2 to $20 \mu \mathrm{m}$, of which a section from 7 to $20 \mu \mathrm{m}$ is shown in each of the images.

and $20 \mu \mathrm{m}$ wide lines can be seen at the imbibition front. These images clearly demonstrate the same shrinkage of the lines upon sintering in the lateral dimensions as demonstrated in earlier papers. ${ }^{18-20}$ While the imbibition front of the PZT lines does appear to recede from their preheat treatment infiltration location, the majority of the shrinkage that occurs in the plane of the substrate is in the transverse direction. The imbibition front recedes approximately $10 \mu \mathrm{m}$ during sintering, but this represents less than $0.5 \%$ of the $2-5 \mathrm{~mm}$ long lines; by comparison, the width of the lines typically decreases by $3-10 \%$. Because the relative shrinkage of the width of the lines is significantly greater than the relative reduction in the length of the lines, the effects of the lengthwise reduction in dimensions are neglected for the purposes of this work.

A similar comparison of AFM images of PZT lines patterned from $5 \mu \mathrm{m}$ wide by $1.85 \mu \mathrm{m}$ high channels is shown in the insets of Figure 1. These images more clearly show that the vertical direction is the axis of greatest shrinkage in the PZT lines because there is no substrate to restrict the contraction of material in this direction. These images also demonstrate the development of the nonuniform "double-peak" profile in which the edges of the lines have greater heights than the interior region, which is discussed in more detail in a subsequent section.

The amount of volumetric shrinkage was estimated by using computer software (Global Lab Image 2, Data Translation, Marlboro, MA) to measure the cross-sectional area of the patterned lines from images such as the one shown in Figure 1A. Figure 3 summarizes the evolution of the film density for the PZT lines patterned from $1.4 \mu \mathrm{m}$ high channels for slow and fast drying rates. Initially, the sol-gel contains approximately $18 \%$ solids. During drying, the gel shrinks to 


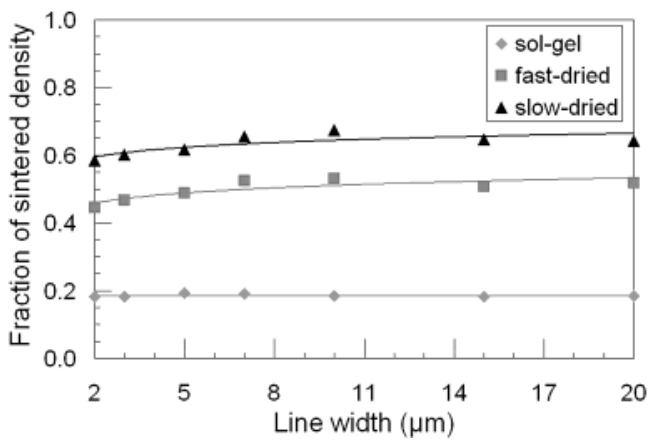

Figure 3. The evolution of gel densities during drying and heat treatment. The sol-gel begins with approximately $18 \%$ solids. Fast drying results in a gel that contains $45-53 \%$ solids, while slow drying produces gels with $58-68 \%$ of the final density. After sintering, the gels have the same final density but differ in topography.

$45-53 \%$ of the final density when dried quickly in air but densifies further to $58-68 \%$ solids when dried more slowly. Water and organics diffuse through the PZT gel network to the exterior surfaces of the line bounded by the PDMS and are then transported through the permeable mold. ${ }^{9}$ As a result, a concentration profile develops within the cross section of the PZT film whereby the interior has a higher concentration of water and organics than the exterior. ${ }^{27,28}$ Eventually, the exterior of the film reaches a low enough water and organic concentration and forms a gel film of high elastic modulus that resists further shrinkage. Stangle and Aksay ${ }^{28}$ developed a theoretical model to calculate the concentration profiles of porous bodies experiencing simultaneous momentum, heat, and mass transfer. Their results indicate that a slow-drying body held at isothermal conditions will have a relatively flat concentration profile compared to a much more pronounced concentration gradient of water and organics for a fast-dried body. As a result, the rigid outer gel layer forms sooner with the faster dried films at a time when the interior of the film contains additional moisture and organics, leading to the measurements of lower density after drying.

The data in Figure 3 also indicate a decreasing trend of volumetric loss during drying with decreasing line widths. As the line width decreases, the ratio of surface area to volume increases. The increased relative surface area of the thinner lines acts as additional scaffolding to counteract the shrinkage stresses, resulting in a less-dense dried film. The additional porosity of the thinner lines is removed upon densification during heat treatment, and the final overall measured volumetric shrinkage is nearly constant across all line widths.

Upon heat treatment, films patterned under either drying condition will shrink to the same overall volume. The less-dense, fast-dried films shrink by a greater amount during heat treatment to remove the porosity. While the final density of the film is unaffected, the drying profile can contribute to two sources of defects in the films: cracking of the patterned features and alteration of the film topography.

B. Cracking. Cracking is a phenomenon that develops during the drying and sintering of the gelled film as additional shrinkage takes place in a nonpliable solid material. In the case of the sintering of patterned PZT sol-gel films, our experience has shown that the development of cracks appears to be a complex function of the following variables: (i) height and width of the patterned lines, (ii) aging of the sol-gel prior to deposition, and (iii) drying rate.

Beh et al. ${ }^{18}$ showed that $\mathrm{ZrO}_{2}$ lines patterned by MIMIC with molds having $2 \mu \mathrm{m}$ depth channels will crack for line widths

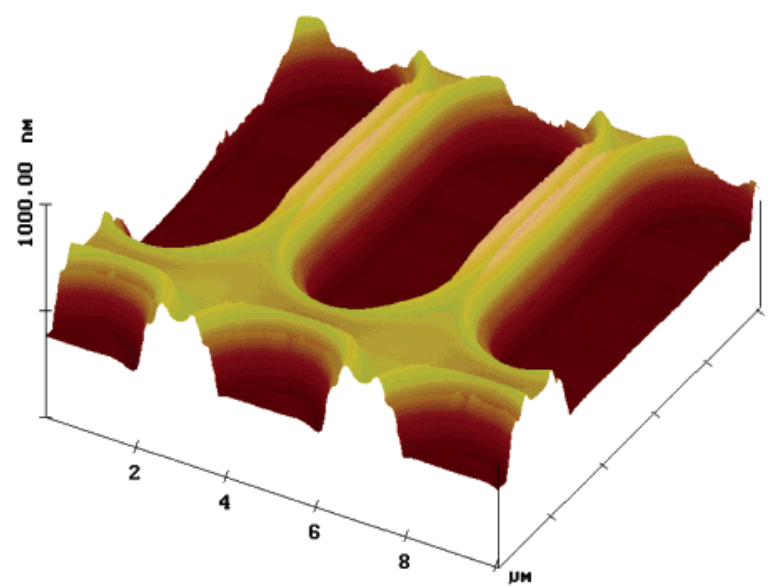

Figure 4. AFM contact mode image of a grid pattern of $2 \mu \mathrm{m}$ wide sintered PZT lines patterned from a $1.85 \mu \mathrm{m}$ depth mold.

with lateral dimensions greater than $14 \mu \mathrm{m}$. We speculate that there is an inverse relation between the critical widths and heights for crack-free lines that can be patterned for different materials via MIMIC. As the channel height is increased, the critical width for crack-free lines is reduced because the additional vertical shrinkage that must occur during sintering for thicker lines must be compensated by a lower stress loading in the lateral dimensions. ${ }^{29,30}$ Variations in the processing of the PZT gels hindered attempts to test and quantify this effect. One potential variation is the degree of aging of the sol-gel prior to deposition; it was observed that there is a reduced propensity for cracking with sol-gels that have been aged for multiple days in sealed vials prior to deposition. These sols have higher viscosities due to the increased cross-linking of the metal-oxygen oligomeric chains reducing the distance that solgel infiltrates the channels during MIMIC. During the additional aging, the organic alkoxide groups of the starting materials have time to disassociate from the metal-oxygen chains, allowing them to be more readily removed during drying and sintering.

More pertinent to the subject of this paper is the relationship between the drying rate and the propensity of cracking. In general, slow-dried samples showed little or no cracking, even for lines as wide as $20 \mu \mathrm{m}$ produced from the $1.85 \mu \mathrm{m}$ depth channels. Meanwhile, many of the fast-dried samples had significant cracking for line widths of $5 \mu \mathrm{m}$. The concentration gradient of the dried film ${ }^{27}$ has produced a gradient of the elastic modulus across the thickness, the interior having a lower modulus than the exterior. If the film is thick enough, the large volumetric shrinkages result in the cracking of the patterned lines. In the fast-dried situation, both the heat-treatment shrinkage requirements and the elastic modulus variation are greater, resulting in increased stresses that lead to an earlier onset of cracking. Our unpublished experimental results from samples patterned from other channel widths and deposited on other substrates also support the notion that slower drying rates reduce the propensity for cracking in the patterned thin films.

C. Double-Peak Topographies. The evolution of the doublepeak topography in the patterned thin films is consistent with the published AFM images presented in earlier papers. ${ }^{18,19}$ The presence of these peaks can pose significant hindrances to the deposition of succeeding layers in device fabrication. The peaks also occur in more complicated geometries than straight lines, as shown in the grid pattern in Figure 4. The existence of peak formation is validated in the SEM images of a cross-sectioned PZT line in Figure 5. It is important to note that the presence of troughs between the peak regions is not due to "buckling" 

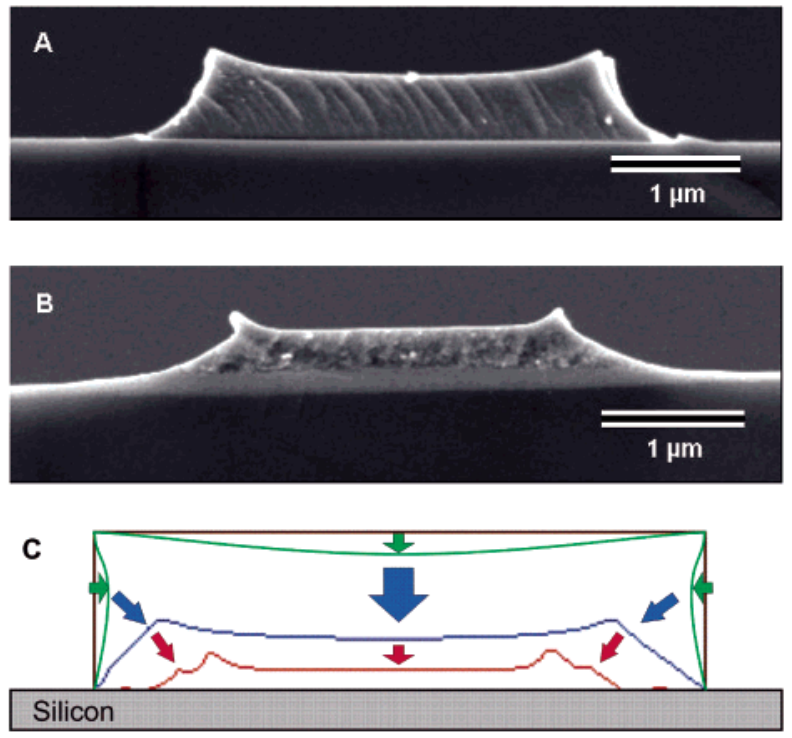

Figure 5. SEM images of a cross-sectioned $5 \mu \mathrm{m}$ wide sintered PZT line on Si patterned from a $1.85 \mu \mathrm{m}$ depth PDMS mold (A) after drying and (B) after heat treatment and (C) a schematic diagram of the drying process leading to peak formation. The black line represents the original contours of the rectangular capillary channel formed by the PDMS mold. During early gelation, the drying film debonds from the flat PDMS sidewalls while the corners continue to wet (green). As the film continues to dry, the shape of the corners is locked-in by the increased evaporation rate (blue). Heat treatment fails to remove the peaks, instead increasing the relative size of the protrusions for fast-dried films (red). The dried and sintered profiles that are shown are generated from actual contact-mode AFM topography data for a fast-dried $5 \mu \mathrm{m}$ wide PZT line.

of the PDMS molds. The PDMS molds used in this study are not of high enough aspect ratio or thin enough to experience any sort of sagging of the tops of the capillary channels. ${ }^{9}$ If sagging did occur for these mold geometries, the double-peak topography would have been observed with all materials patterned in previous studies, such as polymers and colloidal crystals, and not merely ceramic materials derived from solgels that exhibit significant volumetric shrinkage upon drying and sintering.

The difference in the height of the peaks and the bottom of the trough was measured from the AFM data to quantify the peak formation. Figure 6A summarizes the trough height for films that were fast- and slow-dried for different width lines. The trough height increased with line width because the effects of the pinned edges were reduced for the wider lines. The trend asymptotically approaches a limiting value as the shrinkage behavior in the middle of the film approaches that of largearea continuous thin films. The patterned films that dried faster had smaller trough heights than the slow-dried heights after the drying step. The increased rate of evaporation throughout the bulk of the film results in the onset of rigid skin formation on the flat surfaces at a time closer to the onset of skin formation at the corners. As a result, the interior of the film has not densified as much as in the slow-dried films, leading to a lower measured trough height.

After heat treatment, the troughs for the fast-dried films increase in height, while those for the slow-dried films decrease, as shown in Figure 6B. The same rapid film formation that is responsible for the smaller difference in the trough height prior to heat treatment is the cause of the increased trough to peak differential afterward. For the slow-dried films, the majority of the shrinkage during sintering is due to densification of the gel
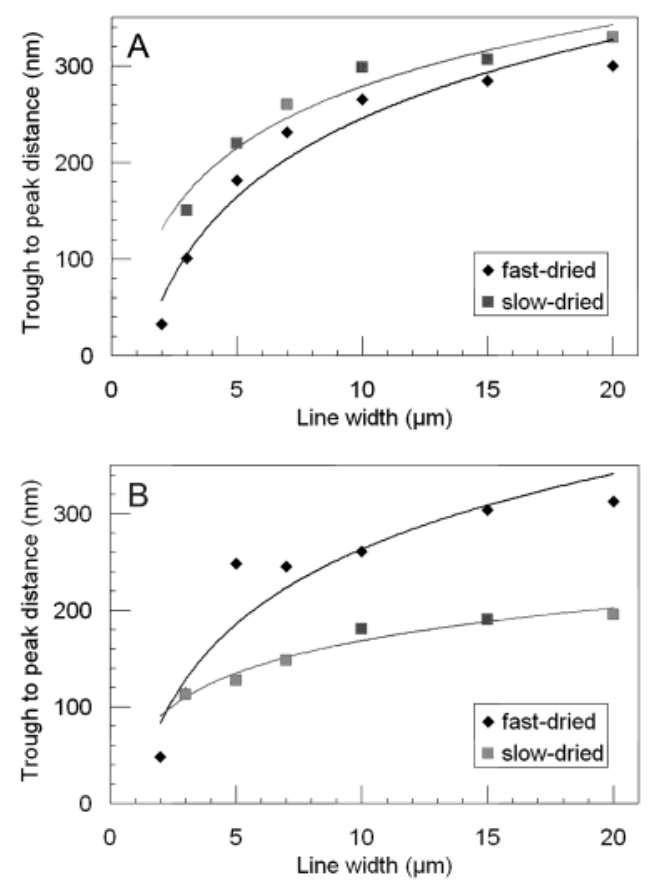

Figure 6. Trough to peak height (A) after drying and (B) after heat treatment at $600{ }^{\circ} \mathrm{C}$ for $3 \mathrm{~h}$ as a function of line width for slow- and fast-dried films.

as the perovskite grain structure evolves. The entire film profile shrinks by approximately the same proportions, so the depth of the trough appears to decrease when the peaks are actually shrinking at the same rate as the interior of the film. AFM measurements indicate that the peak heights decrease by $32-$ $44 \%$ for the slow-dried films, while the film thickness at the trough is reduced by $33-46 \%$. The increased porosity at the interior of the fast-dried thin films is removed during heat treatment, leading to the observation of amplified shrinkage in the trough regions. AFM measurements reveal that the peak heights decrease by $32-42 \%$ for the fast-dried films, which is comparable to the values for the slow-dried films. The dissimilarity lies in the reduction of the substrate to trough distance by $52-60 \%$ for the fast-dried patterns, resulting in the observed increase in trough heights.

The propensity of the patterned thin films to form double peaks can be minimized by drying the thin films more slowly during the gelation and imbibition process. However, the question remains of how to eliminate the characteristic doublepeak profiles entirely. Because the peaks are being formed by the presence of the sharp corners of the PDMS mold, a series of PZT lines were patterned from PDMS molds having no sharp corners. These molds were cast against lithographic masters of patterned photoresist that were baked longer than usual at a temperature above the glass-transition temperature after development to round out the corners. Figure 7 shows a pair of SEM images of PDMS molds mounted in cross section to show how the rounded photoresist produces corner-free channels with depth of $1.4 \mu \mathrm{m}$.

As the AFM traces in Figure 8 indicate, the lack of sharp corners entirely eliminates the formation of peaks in the patterned film. The images include an overlay of the topography of the original PDMS mold to demonstrate the fidelity with which the features of the mold are replicated in the dried and sintered films. To further illustrate the point, a $20 \mu \mathrm{m}$ wide lithographic master was produced with rounded corners and a small "hill" feature down the middle of the channel. This feature 

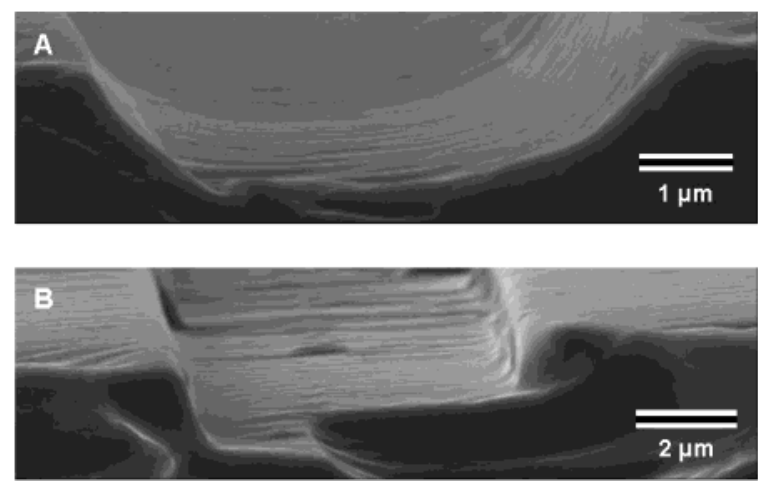

Figure 7. SEM images of cross-sectioned PDMS molds patterned from lithographic master with (A) rounded edges and maximum depth of $1.4 \mu \mathrm{m}$ and (B) sharp corners and depth of $1.85 \mu \mathrm{m}$.
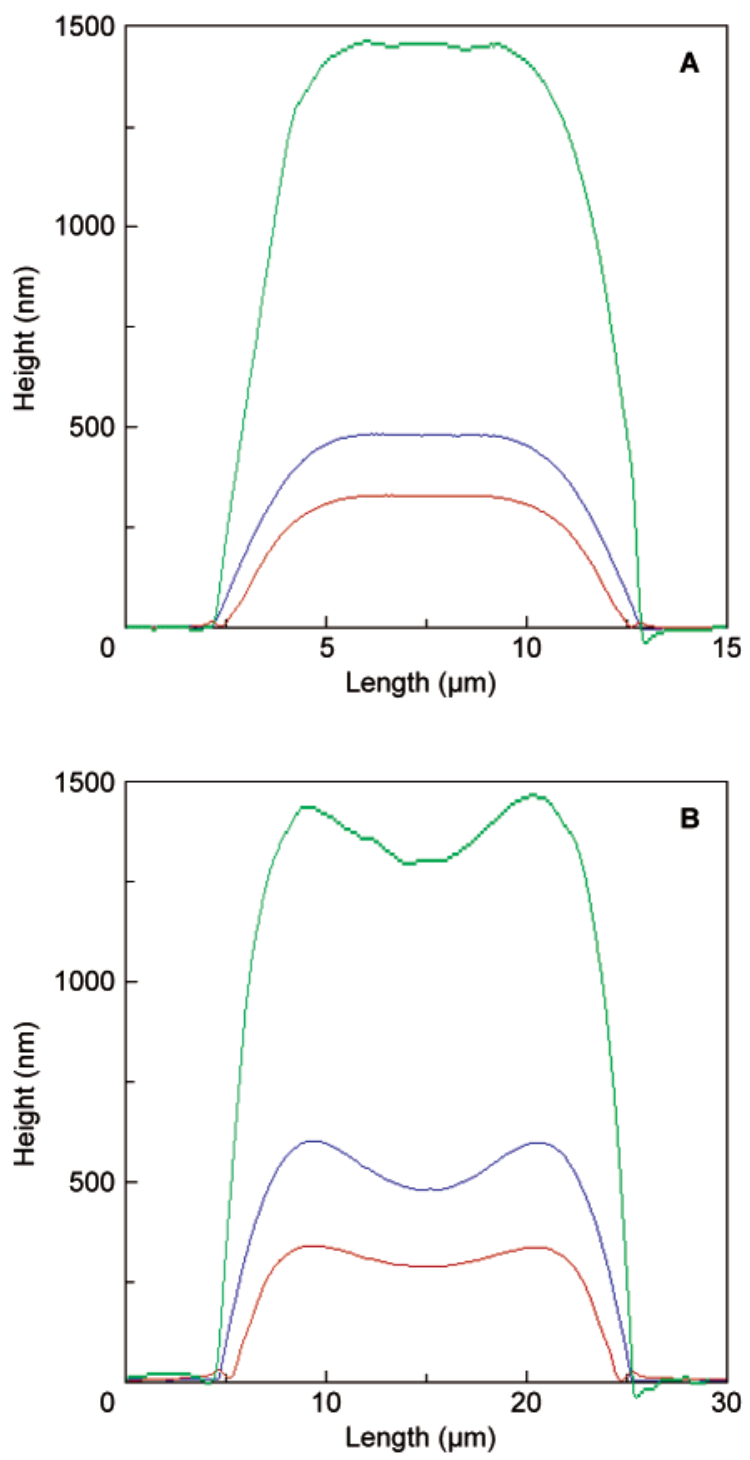

Figure 8. AFM traces showing cross-sectioned view of the pre- (blue) and postheat treatment (red) PZT lines using rounded PDMS mold of $1.4 \mu \mathrm{m}$ depth (green): (A) $10 \mu \mathrm{m}$ wide line dried slowly; (B) $20 \mu \mathrm{m}$ wide line dried rapidly.

was captured in the PDMS mold upon casting and then reproduced in the same proportions in the PZT thin film, as shown in Figure 8B. The film reproduces the topography of the mold regardless of fast or slow drying conditions; however, the drying speed is still important for controlling the propensity for cracking during sintering and the maximum imbibition distance in the capillaries.

D. Theory. The results with the rounded capillary channels clearly demonstrate that the double-peak profile is the direct result of the presence of sharp corners in the rectangular capillary channels. Two phenomena work in concert with one another to effect the final nonuniform topography: (i) preferential wetting of the sol-gel and (ii) increased rate of sol-gel drying at the capillary corners.

1. Preferential Wetting of Sol-Gel to Capillary Corners. Studies of the imbibition of wetting fluids in noncircular capillaries have demonstrated the preference of fluids to wick along the corners over the sides. ${ }^{31,32}$ This effect is thermodynamically driven by the minimization of total surface energies that drive the fluid to adopt rounded and circular profiles. The fluid preferentially wets to zones of small radii of curvature, an effect similar to capillary condensation. The smaller the radius of curvature is, the greater the localized pressure becomes and the thicker the layer of fluid that covers the surface becomes. In the case of capillary channels with rectangular cross section, the fluid preferentially adheres to the corners the radius of curvature of which is very small compared to the flat surfaces that have an infinite radius of curvature. ${ }^{33}$ This effect should not be confused by the distinct phenomenon that drives the actual fluid flow that fills the channels; the flow is driven by the relationship between the curvature of a liquid-vapor interface and local pressure described by Laplace's equation. ${ }^{34}$ A gradient of decreasing capillary pressure is induced by the increasing radius of curvature of the liquid-vapor meniscus along the flow direction. ${ }^{31}$

The shape of the imbibition front is made more complicated by the difference in surface energies of the three PDMS walls compared to the Si substrate. ${ }^{31,35}$ Goniometric measurements show that the contact angle of the PZT sol-gel is $46^{\circ} \pm 3^{\circ}$ on PDMS and $24^{\circ} \pm 2^{\circ}$ on $\mathrm{Si}^{36}$ As a result, the sol-gel preferentially contacts the Si substrate to the walls of the PDMS mold. Thus, because of the stronger adhesion of the sol-gel to the $\mathrm{Si}$, the fluid prefers to wet the bottom corners of the channels bound by the substrate and the PDMS over the all-PDMS corners of the mold. This results in the imbibition front along the Si/PDMS corners stretching further ahead than for the corners formed by the PDMS mold alone, as depicted in Figure 9.

As the gelled PZT shrinks due to the evaporation of water and other volatile organic components, certain surfaces of the capillary channels once again become exposed as the gel debonds from the surface. The regions that de-adhere from the surface first are the areas that were the least favorable for wetting or the surfaces of lowest surface energy. An analogy is that this process is the reverse of the melting of a nonspherical particle whereby the surface liquid prefers to collect in the areas of high curvature on the particle. ${ }^{37}$ In this case, the gel debonds from the PDMS walls first and remains adhered to the substrate. Similarily, because of the preferential wetting of the corners over the straight sides in the rectangular channels, the gel debonds from the flat PDMS surfaces prior to the corners. The side bounded by the two all-PDMS corners is also the longest side due to the rectangular shape of the channel, so this side is the most favorable for debonding of the gel. The result is that the shrinking gel forms a concave shape after the side debonds from the sidewall, but the corners are still in contact.

2. Faster Drying at Capillary Corners. The evaporation of water and organic components occurs fastest at the corners 

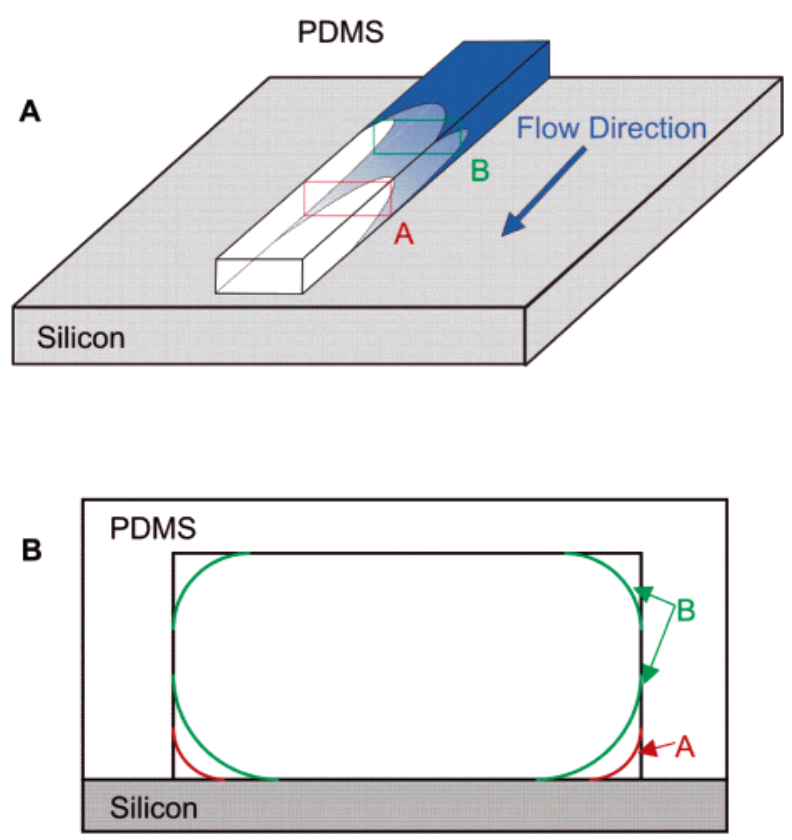

Figure 9. The imbibition front of the PZT sol-gel, which is led by the wetting along the corners of the rectangular capillary channel. Because the PZT sol-gel wets the silicon preferentially over the PDMS, the leading edges of the front are longer along the Si/PDMS corners on the bottom over the PDMS corners at the top (after Dong and Chatzis $^{31}$ ).

because of the higher surface area-to-volume ratio. This is validated using a two-dimensional, transient mass transfer equation to model the evaporation of fluid from a solid cross section:

$$
\frac{\partial C}{\partial t}=D\left(\frac{\partial^{2} C}{\partial x^{2}}+\frac{\partial^{2} C}{\partial y^{2}}\right)
$$

where $C(x, y, t)$ is the concentration, $D$ is the diffusion coefficent, $t$ is time, $x$ is the position along the width of the channel, and $y$ is the coordinate in the height direction. For the purpose of simplification, it is assumed that all of the water and organic components that are volatilized during drying can be treated as a single component with one diffusion coefficient. The equation is nondimensionalized to

$$
\frac{\partial \theta}{\partial \tau}=\frac{\partial^{2} \theta}{\partial X^{2}}+\frac{\partial^{2} \theta}{\partial Y^{2}}
$$

after defining the following variables:

$$
\begin{gathered}
\theta(X, Y, \tau) \equiv \frac{C}{C_{0}} \\
X \equiv \frac{x}{H} \\
Y \equiv \frac{y}{H} \\
\tau \equiv \frac{t H^{2}}{D}
\end{gathered}
$$

where $C_{0}$ is the initial concentration and $H$ is the channel height. The following boundary conditions are imposed to model the
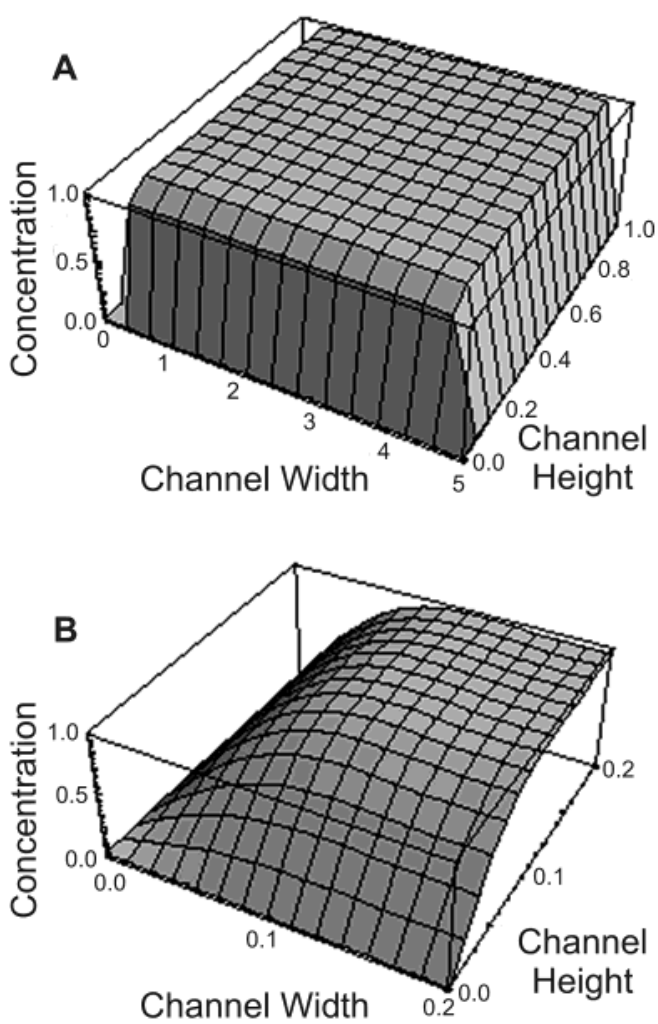

Figure 10. Concentration of water and organic material remaining in the drying sol-gel in a PDMS capillary channel of $\beta=5$ at $\tau=0.001$ for (A) the entire channel cross section and (B) a close-up of the PDMS corner region.

system at early times when the volumetric shrinkage is negligible and the gel boundaries are still in contact with the PDMS mold:

$$
\begin{gathered}
\theta(X, Y, 0)=1 \quad \text { (initial condition) } \\
\frac{\partial \theta}{\partial Y}(X, 1, \tau)=0 \quad(\text { no penetration at substrate surface) } \\
\theta(0, Y, \tau)=\theta(\beta, Y, \tau)=\theta(X, 0, \tau)=0
\end{gathered}
$$

where $\beta$ is the aspect ratio of the channel $(\beta=W / H)$. The boundary conditions in eq 9 assume that the diffusion of the water and organic material through the PDMS mold is infinitely fast compared to the diffusion of the material through the solgel network because of the permeability of PDMS membranes to gaseous species. ${ }^{9}$ For the purposes of this analysis for early times when the PDMS membrane is well below its saturation limit of water and organic content, this assumption is sufficient.

The mass transfer equation was solved analytically using the finite Fourier transform method. ${ }^{38-40}$ The result may be expressed as

$$
\begin{aligned}
\theta(X, Y, \tau) & =\frac{4}{\pi^{2}} \sum_{n=1}^{\infty} \sum_{m=1}^{\infty} \frac{\left(1-(-1)^{n}\right)}{n\left(m-\frac{1}{2}\right)} \times \\
e^{-\pi^{2}\left[(n / \beta)^{2}+(m-(1 / 2))^{2}\right] \tau} & \sin \left(\frac{n \pi X}{\beta}\right) \sin \left[\left(m-\frac{1}{2}\right) \pi Y\right] .
\end{aligned}
$$

Because the solution is only valid at early times prior to the debonding of the drying gel from the channel walls, the plots shown in Figure 10 are at $\tau=0.001$. Figure 10A is a three- 
dimensional plot that describes the residual water and organic concentration over the channel cross section the width of which is five times the channel height. Figure 10B is a close-up of the corner that illustrates how much faster the corner region dries with respect to both the flat surfaces and bulk regions of the capillary channel. By taking the integral of our solution, we estimate that the corner region, defined by an area corresponding to $0.01 \%$ of the total channel cross section, evaporates 12 times faster than a comparable area along the flat edge at the top of the channel.

As drying and gelation occur, the gel increases in rigidity. ${ }^{28}$ The amplified rate of evaporation at the corners results in the development of a more densified gel layer at the corners compared to the flat surfaces and bulk of the film. This region of greater elastic modulus serves to lock in the shape of the corner as the rest of film continues to dry. As the mass of the film dries enough so that all three sides originally bounded by the PDMS walls have debonded, this locked-in shape of the corners leads to the formation of the double-peak profile. Upon further shrinkage during heat treatment, the double-peak profile remains as a remnant of the early drying process. This process is summarized in the schematic diagram in Figure 5C.

\section{Conclusions}

PZT and other sol-gel-based films patterned by MIMIC in rectangular capillary channels form peaks at the edges of the film during gelation and drying. This phenomenon is initiated by the preferential wetting of the gel to the corners of the PDMS and then locked-in by the accelerated film drying rates at the corners. Films that are dried more slowly have a greater density after drying and prior to heat treatment, allowing them to sinter more evenly and with a lower propensity for cracking.

The use of molds cast against lithographic molds having rounded corners of which the radius of curvature is on the same order as that of the dimensions of the channel is a simple and effective method to eliminate the formation of topographical nonuniformities in the patterned thin films. The lack of sharp corners makes the wetting of the sol-gel to the PDMS more uniform, preventing the initiation of peak formation. In addition, the elimination of corners makes the evaporation rates of water and organics more uniform across the entire surface of the film, preventing the nonsymmetric skin formation that locks in the shape of the corners. This permits the original shape of the mold to be preserved in the patterned film. This simple solution prohibits the direct patterning of sol-gel-derived materials with sharp edges by MIMIC, which may prove to be a limitation in certain potential applications.

Acknowledgment. We gratefully acknowledge the technical assistance of D. M. Dabbs and S. M. Miller, both of Princeton University. T. J. Martin of Sensors Unlimited, Inc. provided the computer software and expertise to design the lithographic masks. S. M. Miller also generously provided the patterned lithographic masters used in this work. We thank M. Apel-Paz and K. T. D. Vanderlick at Princeton University for use of their goniometer. The main portion of this work was supported by NASA Grant NAG-2-1475. Partial support by the NASA University Research, Engineering and Technology Institute on BioInspired Materials (BIMat) under Award No. NCC-1-02037 is also acknowledged.

\section{References and Notes}

(1) Scott, J. F.; Paz de Araujo, C. A. Science 1989, 246, 1400.

(2) Polla, D. L.; Francis, L. F. Annu. Rev. Mater. Sci. 1998, 28, 563.

(3) O'Connor, L. Mech. Eng. 1993, 115, 62.

(4) Rosenman, G.; Shur, D.; Skliar, A. J. Appl. Phys. 1996, 79, 7401.

(5) Scott, W. B. Aviat. Week Space Technol. 1993, 138, 36.

(6) Campbell, S. A. The Science and Engineering of Microelectronic Fabrication; Oxford: New York, 1996; p 6.

(7) Jeon, N. L.; Clem, P. G.; Payne, D. A.; Nuzzo, R. G. Langmuir 1996, 12,5350 .

(8) Payne, D. A.; Clem, P. G. J. Electroceram. 1999, 3, 163

(9) Xia, Y.; Whitesides, G. M. Annu. Rev. Mater. Sci. 1998, 28, 153.

(10) Jeon, N. L.; Clem, P. G.; Nuzzo, R. G.; Payne, D. A. J. Mater. Res. 1995, 10, 2996.

(11) Clem, P. G.; Jeon, N.-L.; Nuzzo, R. G.; Payne, D. A. J. Am. Ceram. Soc. 1997, 80, 2821.

(12) Jeon, N. L.; Clem, P.; Jung, D. Y.; Lin, W.; Girolami, G. S.; Payne, D. A.; Nuzzo, R. G. Adv. Mater. 1997, 9, 891.

(13) Zhao, X.-M.; Xia, Y.; Whitesides, G. M. Adv. Mater. 1996, 8, 837.

(14) Kim, J. H.; Lange, F. F.; Cheon, C.-I. J. Mater. Res. 1999, 14, 1194.

(15) Moran, P. M.; Lange, F. F. Appl. Phys. Lett. 1999, 74, 1332.

(16) Kim, E.; Xia, Y.; Whitesides, G. M. J. Am. Chem. Soc. 1996, 118, 5722 .

(17) Trau, M.; Yao, N.; Kim, E.; Xia, Y.; Whitesides, G. M.; Aksay, I. A. Nature 1997, 390, 674.

(18) Beh, W. S.; Xia, Y.; Qin, D. J. Mater. Res. 1999, 14, 3995.

(19) Seraji, S.; Wu, Y.; Jewell-Larson, N. E.; Forbess, M. J.; Limmer, S. J.; Chou, T. P.; Cao, G. Adv. Mater. 2000, 12, 1421.

(20) Vartuli, J. S.; Özenbaş, M.; Chun, C.-M.; Trau, M.; Aksay, I. A. J. Mater. Res. 2003, 18, 1259.

(21) Kumar, A.; Whitesides, G. M. Appl. Phys. Lett. 1993, 63, 2002.

(22) Bechinger, C.; Muffler, H.; Schäfle, C.; Sundberg, O.; Leiderer, P. Thin Solid Films 2000, 366, 135.

(23) Kim, E.; Xia, Y.; Whitesides, G. M. J. Am. Chem. Soc. 1996, 118, 5722 .

(24) Kim, E.; Xia, Y.; Whitesides, G. M. Nature 1995, 376, 581.

(25) Xia, Y.; Kim, E.; Whitesides, G. M. Chem. Mater. 1996, 8, 1558.

(26) Yi, G.; Wu, Z.; Sayer, M. J. Appl. Phys. 1988, 64, 2717.

(27) Scherer, G. W. J. Am. Ceram. Soc. 1990, 73, 3.

(28) Stangle, G. C.; Aksay, I. A. Chem. Eng. Sci. 1990, 45, 1719

(29) Hutchinson, J. W.; Suo, Z. Adv. Appl. Mech. 1992, 29, 63.

(30) Lange, F. F. Science 1996, 273, 903.

(31) Dong, M.; Chatzis, I. J. Colloid Interface Sci. 1995, 172, 278.

(32) Legait, B. J. Colloid Interface Sci. 1983, 96, 28. Ransohoff, T. C.; Radke, C. J. J. Colloid Interface Sci. 1988, 121, 392. Mason, G.; Morrow,

N. R. J. Colloid Interface Sci. 1991, 141, 262.

(33) Celestini, F. Phys. Lett. A 1997, 228, 84.

(34) Adamson, A. W.; Gast, A. P. Physical Chemistry of Surfaces, $6^{\text {th }}$ ed.; Wiley: New York, 1997; pp 4-16.

(35) Kim, E.; Whitesides, G. M. J. Phys. Chem. B 1997, 101, 855

(36) The contact angles of the PZT sol-gel with silicon and PDMS did not vary with the age of the sol-gel. These measurements were repeated on sol-gels that ranged in age from 30 min to 5 months, and no variation was found.

(37) Celestini, F.; Ten Bosch, A. Phys. Lett. A 1995, 207, 307.

(38) Carslaw, H. S.; Jaeger, J. C. Conduction of Heat in Solids, 2nd ed.; Clarendon: Oxford, U.K., 1959.

(39) Butkov, E. Mathematical Physics; Addison-Wesley: Reading, MA, 1968.

(40) Deen, W. M. Analysis of Transport Phenomena; Oxford: New York, 1998; pp 132-194. 